\title{
Essential Laminations in Surgered 3-Manifolds
}

\author{
Ying-Qing Wu
}

Suppose $M$ is a 3-manifold with torus $T$ as a boundary component, and let $P$ be an incompressible surface on $\partial M$ disjoint from $T$. It was proved in [9] that in most cases, $P$ remains incompressible in most of the Dehn filled manifolds $M(\gamma)$. (See Proposition 2 below). The present note is to solve a problem posed informally by Peter Shalen, which asks whether a similar result holds for essential laminations in 3-manifolds. The answer is positive: Essential laminations disjoint from $T$ will usually remain essential after Dehn fillings. Note that the essentiality of a lamination concerns not only compressibility, but also reducibility and existence of end compressing disks in the resulting manifolds.

Throughout this paper, all 3-manifolds are assumed orientable. Let $M$ be a 3-manifold, and $T$ a torus component of the boundary of $M$. For a slope $\gamma$ on $T$, let $M(\gamma)$ be the manifold obtained by attaching a solid torus $V$ to $T$ such that $\gamma$ corresponds to the meridian slope of $V$. If $\gamma_{1}, \gamma_{2}$ are two slopes on $T$, denote their geometric intersection number by $\Delta=\Delta\left(\gamma_{1}, \gamma_{2}\right)$. We refer the readers to [4] for the definition of essential laminations and related notions like branched surfaces, horizontal boundary, vertical boundary, monogons, etc.

Now suppose $M$ is compact, and let $\lambda$ be an essential lamination in $M$. We assume that $\lambda$ is disjoint from $\partial M$. A set $A$ is called a quasi-annulus if $A$ is the image of a continuous map $f: S^{1} \times I \rightarrow M$, such that $\left.f\right|_{S^{1} \times[0,1)}$ is an embedding, $f\left(S^{1} \times 0\right)=A \cap T$, and $f\left(S^{1} \times 1\right)=A \cap \lambda$. $A$ is incompressible if $f\left(S^{1} \times[0,1)\right)$ is incompressible.

Theorem 1 Suppose $M$ contains no incompressible quasi-annulus from $T$ to $\lambda$. If $\lambda$ is not essential in $M\left(\gamma_{1}\right)$ and $M\left(\gamma_{2}\right)$, then $\Delta\left(\gamma_{1}, \gamma_{2}\right) \leq 1$. Thus $\lambda$ is essential in $M(\gamma)$ for all but at most three slopes $\gamma$.

Remark. Generally, the quasi-annulus in the theorem cannot be replaced by an embedded annulus. For example, let $\lambda$ be a Klein bottle, let $M_{1}$ be a twisted I-bundle over $\lambda$. Let $M_{2}$ be a $(p, q)$ cable space, $q \geq 1$. (See e.g. [6, p.124] for definition.) Gluing $M_{2}$ to $M_{1}$ 
along their boundary so that the $(p, q)$ curve on $\partial M_{2}$ is attached to the boundary of the I-bundle over an orientation-reversing simple closed curve in $\lambda$, we obtain a manifold $M$ with a single torus boundary $T$. There is an incompressible quasi-annulus from $T$ to $\lambda$, and $\lambda$ is inessential in $M(\gamma)$ for infinitely many $\gamma$, but there is no incompressible embedded annulus connecting $T$ to $\lambda$. However, if the leaves of $\lambda$ are all orientable, (or if we replace $\lambda$ by a lamination without isolated leaves, as in [4]), then the quasi-annulus can be chosen to be an embedded annulus.

When $M$ contains a quasi-annulus from $T$ to $\lambda$, a similar result like [1, Thm 2.4.3] holds:

Theorem 2 Suppose $M$ is connected and has an incompressible quasi-annulus from $T$ to $\lambda$ with slope $\gamma_{0}$ in $T$. Then

(a) $\lambda$ is not essential in $M\left(\gamma_{0}\right)$.

(b) If $\lambda$ is not essential in $M\left(\gamma_{1}\right)$ for some $\gamma_{1}$ with $\Delta\left(\gamma_{1}, \gamma_{0}\right)>1$, then either $\lambda$ has a torus leaf $l$ parallel to $T$, or $\lambda$ is a Klein bottle and $M$ is a twisted I-bundle over $\lambda$.

(c) $\lambda$ is essential in $M(\gamma)$ for some $\gamma$ such that $\Delta\left(\gamma, \gamma_{0}\right)=1$ if and only if it is essential in $M(\gamma)$ for all such $\gamma$.

The proof of the theorems needs several recent results. The following is a slightly generalized form of a result of Scharlemann (cf. [8, Thm 6.1]).

Proposition 1 Let $K$ be a knot in an orientable 3-manifold $X$ with $X-K$ irreducible. Let $P$ be a surface on $\partial X$ such that $P$ is compressible in $X$ and is incompressible in $X-K$. If some nontrivial surgery on $K$ produces a reducible manifold, then $K$ is a cable knot, and the surgery slope is that of the cabling annulus.

Proof. We first assume that $X$ is compact. If $\partial X$ is incompressible in $X-K$, the result is part of $[8$, Thm 6.1$]$. In general, choose $B=\cup B_{i}$ to be the union of some disjoint compressing disks of $\partial X$ in $X-K$, so that after cutting $X$ along $B$, the new manifold $X_{1}=X$-Int $(N(B))$ has boundary incompressible in $X_{1}-K$. By the hypothesis, $\partial X$ has a compressing disk $D$ such that $\partial D$ does not bound a disk in $X-K$. Among all such compressing disks, we choose one, say $D_{1}$, which is transverse to $B$ and minimizes $\left|D_{1} \cap B\right|$. 
By a standard cutting and pasting argument, one can show that $\left|D_{1} \cap B\right|=0$. Therefore, $D_{1}$ gives rise to a compressing disc of $\partial X_{1}$ in $X_{1}$. The result is now followed by applying $[8$, Thm 6.1$]$ to $\left(X_{1}, K\right)$.

For the noncompact case, choose a compressing disc $D$ of $P$ in $X$, Let $N(K)$ be a regular neighborhood of $K$. The reducing sphere in the surgered manifold gives rise to a planar surface $Q$ in $X-N(K)$. Let $X^{\prime \prime}$ be a regular neighborhood of $D \cup N(K) \cup Q$, and let $X^{\prime}$ be the union of $X^{\prime \prime}$ with compact components of $X$ - Int $X^{\prime \prime}$. It is easy to see that $X^{\prime}-K$ is irreducible, and $P^{\prime}$, a regular neighborhood of $\partial D$ in $X^{\prime}$, is compressible in $X^{\prime}$ and incompressible in $X^{\prime}-K$. The conclusion now follows from that in compact case.

Let $m$ be the the slope on $\partial N(K)$ represented by a meridian of $N(K)$, and let $\gamma$ be the slope of the cabling annulus. Since $\Delta(m, \gamma)=1$, we have

Corollary 1 Suppose $M$ is an irreducible 3-manifold with torus $T$ as a boundary component, and suppose $P$ is an incompressible surface on $\partial M-T$. If $P$ is compressible in $M\left(\gamma_{1}\right)$, and $M\left(\gamma_{2}\right)$ is reducible, then $\Delta\left(\gamma_{1}, \gamma_{2}\right) \leq 1$.

Proposition 2 ([9]). Let $M$ be a 3-manifold with torus $T$ as a boundary component, let $P$ be an incompressible surface in $\partial M-T$. Suppose there is no incompressible annulus with one boundary component in $P$ and the other in T. If $P$ is compressible in both $M\left(\gamma_{1}\right)$ and $M\left(\gamma_{2}\right)$, then $\Delta\left(\gamma_{1}, \gamma_{2}\right) \leq 1$.

The following result and its proof are also known to Gordon and Luecke. Recently they completely proved the reducibility conjecture: The conclusion in Proposition 3 is true even if $\partial M=T$.

Proposition 3 Let $M$ be an irreducible 3-manifold, and let $T$ be a torus component of $\partial M$. Suppose $\partial M \neq T$. If both $M\left(\gamma_{1}\right)$ and $M\left(\gamma_{2}\right)$ are reducible, then $\Delta\left(\gamma_{1}, \gamma_{2}\right) \leq 1$.

Sketch of Proof. Take an atoroidal decomposition of $M$ and let $N$ be the part containing $T$. Then either $N\left(\gamma_{i}\right)$ is a solid torus or it is reducible. The possibility of both $N\left(\gamma_{i}\right)$ being solid tori can be ruled out by [2, Thm 1.1], [5, Lemma 3.3], and [6] (or [10]). If $\partial N\left(\gamma_{1}\right)$ is compressible and $N\left(\gamma_{2}\right)$ is reducible, the result follows from Scharlemann's Theorem 
(Corollary 1 above). So suppose both $N\left(\gamma_{i}\right)$ are reducible and $\partial$-irreducible. By filling the non-toral components of $\partial N$ with some suitable manifolds (see the proof of $[8$, Thm 6.1]), we may assume that $N$ is irreducible and atoroidal with boundary consists of tori. Furthermore, since $\partial M \neq T$, these manifolds can be chosen so that $\partial N$ consists of at least two tori. By [3, Cor 2.4], $\mathrm{N}$ must be a rational homology cobordism between $T$ and some torus $T^{\prime}$. Thus any reducing sphere of $N\left(\gamma_{i}\right)$ is separating. Attaching a knot complement to $T^{\prime}$, we get a manifold $X$ satisfying (1) $\operatorname{dim} H_{1}(X ; Q)=1$, and (2) for each $i, X\left(\gamma_{i}\right)$ is non prime, and one of its factors contains an incompressible torus (i.e. the $T^{\prime}$ above). According to [1, Thm 2.2.1, Thm 2.3.1], there is a closed surface $S$ in $X$ which is compressible in $X\left(\gamma_{1}\right)$ and incompressible in $X(\gamma)$ whenever $\Delta\left(\gamma_{1}, \gamma\right) \geq 2$. Cutting along $S$ and applying Corollary 1, we conclude that $X\left(\gamma_{2}\right)$ is irreducible unless $\Delta\left(\gamma_{1}, \gamma_{2}\right) \leq 1$.

Proof of Theorem 1. By [4, Prop.4.5], the lamination $\lambda$ is fully carried by some essential branched surface $B$. Let $X$ be the component of $M-\operatorname{Int} N(B)$ containing $T$, and let $S$ be the horizontal surface $\left(\partial_{h} B\right) \cap X$ on $\partial X$. We first consider the case when there is an incompressible annulus $A$ in $X$ with one boundary component $\partial_{1}$ in $T$ and the other $\partial_{2}$ in $S$. Consider the 1 -foliation $v$ on $N(B)$. ( $v$ is an I-bundle over $B$ if $B$ is a surface). Since $\lambda$ is fully carried, each leaf of $v$ will intersect $\lambda$. Suppose $x$ is a point in $\partial_{2}$. Let $I_{x}$ be the leaf in $N(B)$ with $x$ as an endpoint. Because $\lambda$ is a closed set, there is a point $\alpha(x)$ on $I_{x}$ which is the closest to $x$ among all of the point in $\lambda \cap I_{x}$. This defines a map $\alpha: \partial_{2} \rightarrow \lambda$. Since $\lambda$ is transverse to $v$ and $\lambda$ is a closed set, it is easy to see that $\alpha$ is a continuous map. Let $J_{x}$ be the arc in $I_{x}$ between $x$ and $\alpha(x)$. Then $A^{\prime}=\cup\left\{J_{x} \mid x \in \partial_{2}\right\}$ is a quasi-annulus from $\partial_{2}$ to $\alpha\left(\partial_{2}\right) \subset \lambda$. $A^{\prime}$ is embedded unless some points $x, y$ of $\partial_{2}$ are the endpoints of a single leaf $I_{x}=I_{y}$. In this case, when traveling along $\alpha\left(\partial_{2}\right)$ from $\alpha(x)$ to $\alpha(y)$, the leaf of $\lambda$ containing $\alpha\left(\partial_{2}\right)$ will change its orientation and hence is nonorientable. Thus, $A \cup A^{\prime}$ is a quasi-annulus from the curve $\partial_{1}$ in $T$ to the curve $\alpha\left(\partial_{2}\right)$ in $\lambda$. It is incompressible because $A$ is incompressible, and it is an embedded annulus when the leaves of $\lambda$ are orientable.

Now we assume there is no incompressible annulus between $S$ and $T$. We want to show that, for most slopes $\gamma$ on $T$, the branched surface $B$ which fully carries $\lambda$ and is essential in $M$ will remain essential in $M(\gamma)$. Since $\lambda$ is assumed in Int $M$, and since $B$ is essential in $M$, most of the conditions for $B$ to be essential in $M(\gamma)$ are satisfied. The 
only things we need to check are: (1) $X(\gamma)$ is irreducible; (2) $S$ is incompressible in $X(\gamma)$; and $(3) X(\gamma)$ has no monogons. Now we can use the above theorems. Suppose $\lambda$ is not essential in $X\left(\gamma_{1}\right)$ and $X\left(\gamma_{2}\right)$, and suppose $\Delta>1$. Then by Proposition $2, S$ can not be compressible in both $X\left(\gamma_{1}\right)$ and $X\left(\gamma_{2}\right)$; and by Propositions 1 and 3 , if one of the $X\left(\gamma_{i}\right)$ is reducible, then the other one is irreducible and contains no compressing discs of $S$.

The remaining case is that one of the $X\left(\gamma_{i}\right)$, say $X\left(\gamma_{1}\right)$, is irreducible and has $S$ incompressible in it. Then it has a monogon, which by definition is a compressing disc $D$ of $\partial X$ in $X\left(\gamma_{1}\right)$ intersecting the vertical boundary $\partial_{v} N(B)$ in a unique essential arc. Let $A$ be the component of $\partial_{v} N(B)$ intersecting $\partial D$. ( $A$ is an annulus). Let $B$ be the regular neighborhood of $\partial D$ in $\partial X$. Then $A \cup B$ is a once punctured torus. Its boundary is a simple closed curve $\alpha$ lying in $S$, and $\alpha$ bounds a disk $D_{1}$ on the boundary of a regular neighborhood $N$ of $A \cup D$ in $M$. ( $N$ is a solid torus). Since $S$ is incompressible in $X\left(\gamma_{1}\right)$, $\alpha$ bounds a disc $D_{2}$ in $S$. Since $X\left(\gamma_{1}\right)$ is irreducible, $D_{1} \cup D_{2}$ bounds a 3-cell. Therefore $X\left(\gamma_{1}\right)$ is a solid torus, and $S$ is a longitude annulus.

Let $K$ be the central curve of the attached solid torus in $X\left(\gamma_{1}\right)$. Since $X$ is irreducible, $K$ is not contained in a 3-cell. Applying Corollary 1 with $P=\partial X$, we see that $X\left(\gamma_{2}\right)$ is irreducible. Thus either $S$ is compressible in $X\left(\gamma_{2}\right)$, or $X\left(\gamma_{2}\right)$ has a monogon. In both case $X\left(\gamma_{2}\right)$ has compressible boundary, and hence is a solid torus. By Proposition 2, $K$ is isotopic to a curve on $\partial X$, and therefore is some $(p, q)$ cable of the center curve of $X\left(\gamma_{1}\right)$. Since $K$ is not isotopic to a curve on $S$, we have $q>1$. Let $r=\Delta\left(\gamma_{1}, \gamma_{2}\right) \geq 2$, and let $s=\Delta(l, m)$, where $l$, the center curve of $S$, is a longitude of $X\left(\gamma_{1}\right)$, and $m$ is the boundary of a meridian disc of $X\left(\gamma_{2}\right)$. According to [5, lemma 7.2], $s=r p q \pm 1$. Thus $|s|>1$. This is a contradiction because then no meridian disc of $X\left(\gamma_{2}\right)$ could be a monogon or a compressing disc of $S$.

Proof of Theorem 2. One might try to proceed as in the proof of Theorem 1 by considering the surgered manifold $X\left(\gamma_{i}\right)$. But there would be some difficulty in proving (c) because the inessentiality of the branched surface does not imply the inessentiality of the lamination it carries. Thus we prefer to use the definition of essential laminations. Remember that $\lambda$ is essential iff no leaf is a sphere, $M_{\lambda}$ is irreducible, and $\partial M_{\lambda}$ is both incompressible and end incompressible in $M_{\lambda}$, where $M_{\lambda}$ is $M$ "cutting" along $\lambda$. (See [4, 
p45] for details).

The essential quasi-annulus $A$ from $T$ to $\lambda$ induces in $M_{\lambda}$ a quasi-annulus from $T$ to $\partial M_{\lambda}$ which can be modified to an embedded essential annulus, which we will still denote by $A$. This gives rise to a compressing disk of $\partial M_{\lambda}$ in $M_{\lambda}\left(\gamma_{0}\right)$, and (a) follows. The proof of (c) is the same as that of [1, Thm 2.4.3]: $M_{\lambda}\left(\gamma_{1}\right)$ is homeomorphic to $M_{\lambda}\left(\gamma_{2}\right)$ if $\Delta\left(\gamma_{1}, \gamma_{0}\right)=\Delta\left(\gamma_{2}, \gamma_{0}\right)=1$. So they have the same properties.

To prove (b), we notice that the compressing disk in $M_{\lambda}\left(\gamma_{0}\right)$ obtained by gluing a meridian disk to $A$ will intersect the central curve $K$ of the attached solid torus just once. Thus $K$ is not a cable knot. By Proposition $1, M_{\lambda}\left(\gamma_{1}\right)$ is irreducible. If $\partial M_{\lambda}\left(\gamma_{1}\right)$ is end incompressible, then it must be compressible. By [1, Thm 2.4.3(b)], the component of $M_{\lambda}$ containing $T$ is homeomorphic to $T \times I$, and $\partial M_{\lambda}$ is one of the torus components. Since the natural map from $M_{\lambda}$ to $M$ is an immersion, its restriction to $\partial M_{\lambda}$ is a covering map to a leaf $l$ of $\lambda$. If $l$ is a torus, then $T$ is parallel to $l$. Otherwise $l$ is a Klein bottle and $T$ bounds an I-bundle over $l$.

It remains to show that there is no end-compressing disk in $M_{\lambda}(\gamma)$. (Note that $\partial D$ is an arc, not a circle). Suppose $D$ were an end-compressing disc of $\partial M_{\lambda}$ in $M_{\lambda}(\gamma)$ which minimizes $|D \cap T|$. Let $Q$ be the planar surface $D \cap M_{\lambda}$ which is isotoped to minimize $|Q \cap A|$. Then $A \cap Q$ consists of lines connecting inner boundary components of $Q$ to its outer boundary $\partial D$, and each inner boundary component is incident to $\Delta$ such lines. Thus there are at least two pairs of parallel lines. Since $D$ is an end-compressing disc, at least one pair of parallel lines $\left\{l_{1}, l_{2}\right\}$ bounds a compact band in $Q$. That is, there is a disc $B$ in $Q$ such that $\partial B=l_{1} \cup l_{2} \cup b \cup c$, where $b$ is an $\operatorname{arc}$ in $T$ and $c$ is an arc in $\partial D$. Now a regular neighborhood of $(c \cup$ outer boundary of $A$ ) is a punctured torus whose boundary $\alpha$ bounds a disc in $M_{\lambda}$. Since $\partial M_{\lambda}$ is incompressible, $\alpha$ bounds a disc on $\partial M_{\lambda}$. So this boundary component is a closed torus, and hence cannot have any end-compressing disc, a contradiction.

I was recently informed that Hatcher and Oertel [7] have also obtained some result on these problems. They have a simple proof in case there is no essential incompressible surfaces in the component of the complement of the branched surface which contains the torus $T$. 
I am very grateful to Cameron Gordon and John Luecke for many helpful discussions and comments. Thanks also to Peter Shalen for asking the problem in Theorem 1.

University of Texas at Austin, Austin, TX 78712

and

Nanjing Normal University, Nanjing, P.R.CHINA

\section{References}

[1] M. Culler, C. Gordon, J. Luecke and P. Shalen, Dehn surgery on knots, Ann. Math. 125 (1987) 237-300.

[2] D. Gabai, Surgery on knots in solid tori, Topology, 28 (1989) 1-6.

[3] D. Gabai, Foliations and topology of 3-manifolds, J. Diff. Geom., 18 (1983) 445-503.

[4] D. Gabai and U. Oertel, Essential laminations in 3-manifolds, Ann. Math. 130 (1989) $41-73$.

[5] C. Gordon, Dehn surgery and satellite knots, Trans. Amer. Math. Soc. 275 (1983) $687-708$.

[6] C. Gordon, R. Litherland, Incompressible planar surfaces in 3-manifolds, Topology Appl. 18 (1984) 121-144.

[7] A. Hatcher and U. Oertel, Essential laminations and Dehn filling, preprint.

[8] M. Scharlemann, Producing reducible 3-manifolds by surgery on a knot, Topology 29 (1990) 481-500.

[9] Y.Q. Wu, Incompressibility of surfaces in surgered 3-manifold, Topology (to appear).

[10] Y.Q. Wu, On the reducibility of surgered 3-manifolds, Topology and its Appl. (to appear). 